\title{
Multi-watt 589nm fiber laser source
}

J. W. DAWSON, A. D. DROBSHOFF, R. J. BEACH, M. J. MESSERLY, S. A. PAYNE, A. BROWN, D. M. PENNINGTON, D. J. BAMFORD, S. J. SHARPE, D. J. COOK

January 23, 2006

SPIE Photonics West Conference

San Jose, CA, United States

January 21, 2006 through January 26, 2006 
This document was prepared as an account of work sponsored by an agency of the United States Government. Neither the United States Government nor the University of California nor any of their employees, makes any warranty, express or implied, or assumes any legal liability or responsibility for the accuracy, completeness, or usefulness of any information, apparatus, product, or process disclosed, or represents that its use would not infringe privately owned rights. Reference herein to any specific commercial product, process, or service by trade name, trademark, manufacturer, or otherwise, does not necessarily constitute or imply its endorsement, recommendation, or favoring by the United States Government or the University of California. The views and opinions of authors expressed herein do not necessarily state or reflect those of the United States Government or the University of California, and shall not be used for advertising or product endorsement purposes. 


\title{
Multi-watt 589nm fiber laser source
}

\author{
Jay W. Dawson, Alex D. Drobshoff, Raymond J. Beach, Michael J. Messerly, Stephen A. Payne, \\ Aaron Brown, Deanna M. Pennington, Douglas J. Bamford*, Scott J. Sharpe* and David J. Cook* \\ National Ignition Facility, Livermore National Laboratory, 7000 East Avenue, Livermore, CA \\ 94550, *Physical Sciences Incorporated, 2110 Omega Road Suite D, San Ramon, CA \\ Tel: 925-422-1617, FAX: 925-423-6195, e-mail: dawson17@1ln1.gov
}

\begin{abstract}
We have demonstrated $3.5 \mathrm{~W}$ of $589 \mathrm{~nm}$ light from a fiber laser using periodically poled stoichio-metric Lithium Tantalate (PPSLT) as the frequency conversion crystal. The system employs $938 \mathrm{~nm}$ and $1583 \mathrm{~nm}$ fiber lasers, which were sum-frequency mixed in PPSLT to generate 589nm light.
\end{abstract}

The $938 \mathrm{~nm}$ fiber laser consists of a single frequency diode laser master oscillator $(200 \mathrm{~mW})$, which was amplified in two stages to $>15 \mathrm{~W}$ using cladding pumped $\mathrm{Nd}^{3+}$ fiber amplifiers. The fiber amplifiers operate at $938 \mathrm{~nm}$ and minimize amplified spontaneous emission at $1088 \mathrm{~nm}$ by employing a specialty fiber design, which maximizes the core size relative to the cladding diameter. This design allows the 3-level laser system to operate at high inversion, thus making it competitive with the competing $1088 \mathrm{~nm} 4$-level laser transition. At $15 \mathrm{~W}$, the $938 \mathrm{~nm}$ laser has an $\mathrm{M}^{2}$ of 1.1 and good polarization (correctable with a quarter and half wave plate to $>15: 1$ ). The $1583 \mathrm{~nm}$ fiber laser consists of a Koheras $1583 \mathrm{~nm}$ fiber DFB laser that is pre-amplified to $100 \mathrm{~mW}$, phase modulated and then amplified to $14 \mathrm{~W}$ in a commercial IPG fiber amplifier.

As a part of our research efforts we are also investigating pulsed laser formats and power scaling of the $589 \mathrm{~nm}$ system. We will discuss the fiber laser design and operation as well as our results in power scaling at $589 \mathrm{~nm}$.

Keywords: Fiber lasers, neodymium lasers, erbium lasers, sum frequency mixing, non-linear frequency conversion

\section{INTRODUCTION}

High power lasers capable of generating $589.2 \mathrm{~nm}$ light for efficient excitation of the D2 line of the Na atom are of great interest to the astronomical scientific community for their use as laser guide stars in adaptive optics systems. These lasers are focused onto the sodium layer of the atmosphere, which is approximately $10 \mathrm{~km}$ thick and located $90 \mathrm{~km}$ above the surface of the earth. In this layer of the atmosphere there is a relative abundance of alkali vapor particularly $\mathrm{Na}$ vapor. A high power $589.2 \mathrm{~nm}$ laser focused on this layer of the atmosphere generates a spot of light that appears to a telescope as an artificial star. This artificial star can be re-imaged through the telescope onto a Shack-Hartmann wavefront sensor and provides significant details about the atmospheric optical distortion. This information can then be fed back to a deformable mirror in the telescope optical train to correct for the phase errors and significantly improve the resolution of a large telescope. A natural star can also serve this purpose, but suitably bright natural stars occur only within about $10 \%$ of the visible sky. Laser generated guide stars enable expansion of adaptive optics techniques to greater than $60 \%$ of the visible sky.

As a result of the great improvement in telescope imagery from sodium laser guide stars, there has been a great deal of work recently in developing laser based adaptive optic systems. In particular, the Lick, Keck, Gemini North and South, Paranal, Palomar and Subaru observatories are all developing sodium laser guide star adaptive optic systems as well as Starfire Optical Range. A number of laser technologies have been pursued in conjunction with this work. Dye lasers have been engineered to relative robustness and deployed at the Lick and Keck Observatory [1] and one is currently being deployed at the Paranal observatory [2]. Starfire Optical Range has deployed a 50W CW single frequency laser based on sum-frequency mixing (SFM) 1319nm and 1064nm Nd:YAG lasers. ARFL developed this laser using a series of phase locked loops to generate the intense IR light needed for SFM and resonant cavity enhancement increases the 
frequency conversion efficiency in LBO to an acceptable level [3]. Coherent Technologies has developed a CW modelocked laser system based on Nd:YAG lasers for the Gemini North Observatory [4]. The University of Chicago in collaboration with Caltech has developed and deployed a micro-macro pulse Nd:YAG SFM laser system for the Palomar Observatory [5]. However, all of these systems have a high degree of complexity and most require a laser technician to be on-call at the telescope to ensure reliable functioning of the laser. There is a desire for a more robust solution such as would be provided by a suitable fiber laser. A fiber laser master-oscillator power amplifier configuration would also enable the possibility of generating specific pulse formats of interest to the adaptive optics community for gating out lower atmospheric Rayleigh scattering or even tracking the laser pulse through the sodium layer to eliminate spot elongation effects.

Some common requirements for lasers for this application are $>10 \mathrm{~W}$ of diffraction limited output power, wavelength of $589.2 \mathrm{~nm}$ locked to the sodium D2 line, bandwidth $<3 \mathrm{GHz}$ (preferably less than $500 \mathrm{MHz}$ ), which is the Doppler broadened linewidth of $\mathrm{Na}$ in the upper atmosphere. The ability to generate up to $500 \mathrm{MHz}$ is desirable in order to eliminate saturation effects particularly in pulsed laser systems. The ability to run the laser in a pulsed mode is also desirable as discussed above. We are developing fiber laser system to meet these requirements based on sum frequency mixing a $938 \mathrm{~nm} \mathrm{Nd}^{3+}$ fiber laser with a $1583 \mathrm{~nm} \mathrm{Er}^{3+}$ fiber laser [6] (see figure 1 below).

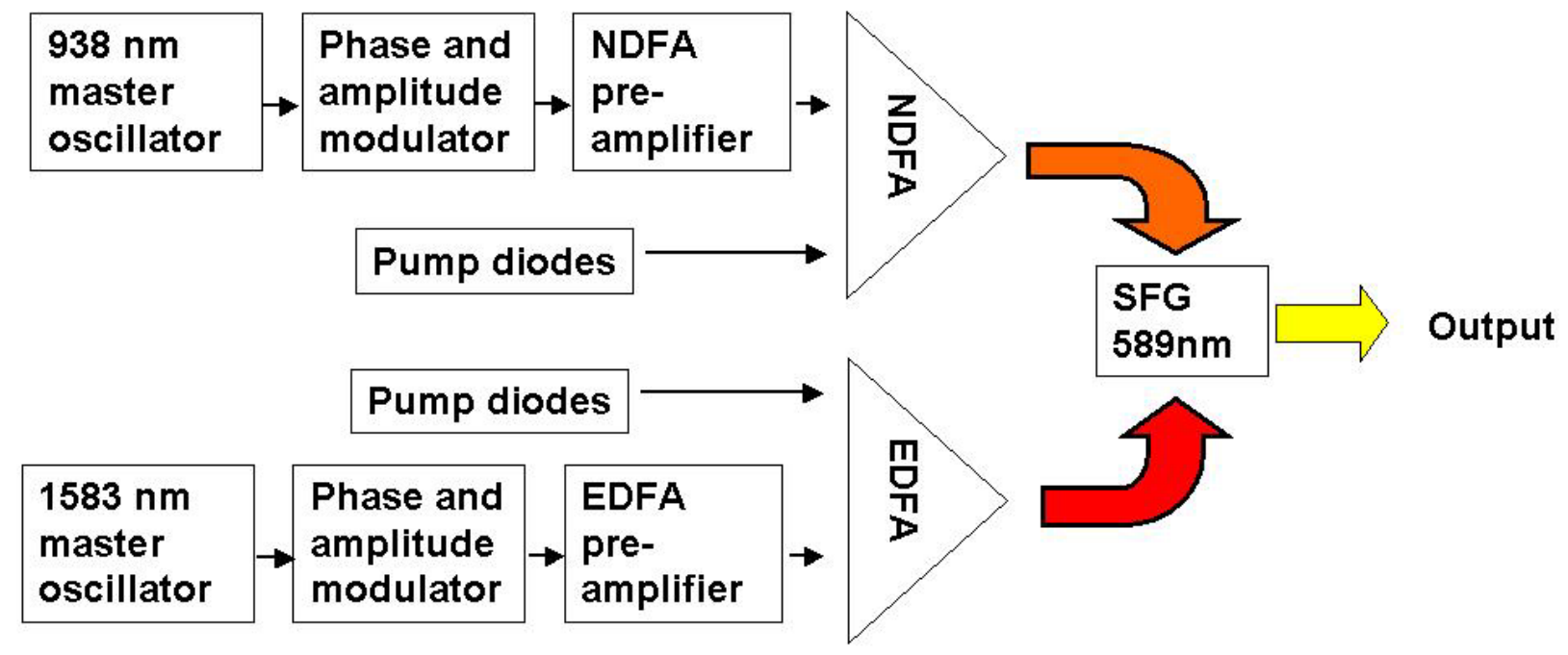

Figure 1: Schematic of the LLNL sum frequency laser for generation of 589nm light guide star applications.

Our system sum frequency mixes $938 \mathrm{~nm}$ light with $1583 \mathrm{~nm}$ light to generate $589 \mathrm{~nm}$ light. A $938 \mathrm{~nm}$ diode laser master oscillator is amplitude and phase modulated and then amplified in a 2-stage neodymium doped fiber amplifier custom designed at LLNL. A fiber DFB laser acts as the $1583 \mathrm{~nm}$ master oscillator, it is phase and amplitude modulated and then amplified in an simple erbium doped fiber preamplifier and an IPG $14 \mathrm{~W}$ power amplifier. The output of these lasers are collimated, combined in a dichroic and focused into periodically poled stoichometric lithium tantalate crystal where they sum-frequency mix to generate $589 \mathrm{~nm}$ light. The $938 \mathrm{~nm}$ and $1583 \mathrm{~nm}$ laser systems are described in detail below along with the sum-frequency mixing scheme.

\section{NEODYMIUM FIBER LASER AT 938NM}

Our 938nm fiber laser master oscillator is an Axcel Photonics 200mW Fabry Perot diode laser mounted in a standard diode laser mount with an integrated bias T. This diode conveniently lases in a single longitudinal mode at $938 \mathrm{~nm}$ with some adjustment of the wavelength possible via temperature control. In a system deployed in the field this would need to be replaced with an external cavity laser diode. The bias $\mathrm{T}$ permits modulation of the laser diode drive current with an RF noise source, which allows us to broaden the laser diode line width to up 500MHz. This line width broadening is simultaneously verified with a grating based optical spectrum analyzer and a Fabry Perot optical spectrum analyzer. The diode laser master oscillator passes through an optical isolator, an acousto-optic modulator and pair of dichroic filters. The dichroic filters pass $938 \mathrm{~nm}$ but reject $1088 \mathrm{~nm}$ and $808 \mathrm{~nm}$ that may be traveling backwards from the first $938 \mathrm{~nm}$ 
fiber amplifier. The light from the diode laser is then coupled through a lens into our $938 \mathrm{~nm}$ neodymium fiber amplifier chain. The amplifier chain consists of a first stage amplifier that brings the signal in the 1-4W range depending upon the duty cycle of the pulse modulation and a power amplifier that brings the final power to $15 \mathrm{~W}$.

Our $938 \mathrm{~nm}$ fiber amplifiers are based on our in house fiber design, which was custom fabricated for us by Nufern. The fiber core composition is critical to good performance at $938 \mathrm{~nm}$. The core consists of silica, neodymium and germania with no other co-dopants. This composition has been shown [7] to provide a significant shift of the standard neodymium emission spectra in glass. The usual $1064 \mathrm{~nm}$ emission peak shifts to $1088 \mathrm{~nm}$ and the usual $915 \mathrm{~nm}$ emission peak shifts to $938 \mathrm{~nm}$. Addition of any phosphorous, aluminum or other co-dopants shifts the spectra back to shorter wavelengths. As we are using the $938 \mathrm{~nm}$ in a sum frequency scheme with $1583 \mathrm{~nm}$ as the other wavelength, wavelengths shorter than $938 \mathrm{~nm}$ require a second wavelength beyond the reach of the erbium amplifier gain bandwidth. However, the use of this core composition strictly limits the doping concentration of $\mathrm{Nd}^{3+}$ in the glass to $<10 \mathrm{~dB} / \mathrm{m}$ of absorption at $808 \mathrm{~nm}$. For our narrow line width, high power application, we require a relatively short fiber amplifier with a large core area that is capable of output powers in the $20 \mathrm{~W}$ range at $938 \mathrm{~nm}$ with suppression of amplified spontaneous emission and parasitic laser action at $1088 \mathrm{~nm}$. We solved this problem by making the area of the fiber core large relative to the area of the pump cladding [8].

The first stage amplifier has a $20 \mu \mathrm{m}$ diameter core with a $0.06 \mathrm{NA}$. The fiber pump cladding was a $125 \mu \mathrm{m}$ in diameter and the fiber is $25 \mathrm{~m}$ long. The fiber was pumped by $35 \mathrm{~W}$ of $808 \mathrm{~nm}$ light that counter-propagated relative to the signal. The $808 \mathrm{~nm}$ light was coupled through a pair of dichorics that protected the pump laser from $938 \mathrm{~nm}$ light and $1088 \mathrm{~nm}$ light that might be generated by a Q-switch resulting from the inadvertent loss of the $938 \mathrm{~nm}$ seed beam. The fiber was coiled to an $8 \mathrm{~cm}$ diameter in a single layer and taped inside a water-cooled copper tube. The purpose of this tube was to ensure the fiber was maintained at a constant temperature close to that of the laboratory. $\mathrm{Nd}^{3+}$ at $938 \mathrm{~nm}$ has a power conversion efficiency in the fiber amplifier of the pump laser power to signal laser power in the $10-30 \%$ range. This results in significant amounts of heat being dumped into the fiber itself. The gain dynamics of the amplifier are sensitive to the fiber temperature. The $938 \mathrm{~nm}$ transition is a 3-level transition the gain at that wavelength is affected by the absorption cross-section of the unexcited ion at $938 \mathrm{~nm}$. The $1088 \mathrm{~nm}$ transition is a 4-level transition for which there is no ground state absorption. Figure 2 (LHS) shows the $938 \mathrm{~nm}$ absorption cross section of the $\mathrm{Nd}^{3+}$ ion in our glass composition. The models from which we designed our fiber assume the fiber is at room temperature. At higher temperatures, the absorption cross-section increases rapidly resulting in much more $1088 \mathrm{~nm}$ gain for the same gain at $938 \mathrm{~nm}$. Figure 2 (RHS) shows the effect of changing the temperature of the copper heat sink for the fiber coil. It appears that lowering the temperature somewhat results in a significant efficiency increase at $938 \mathrm{~nm}$.
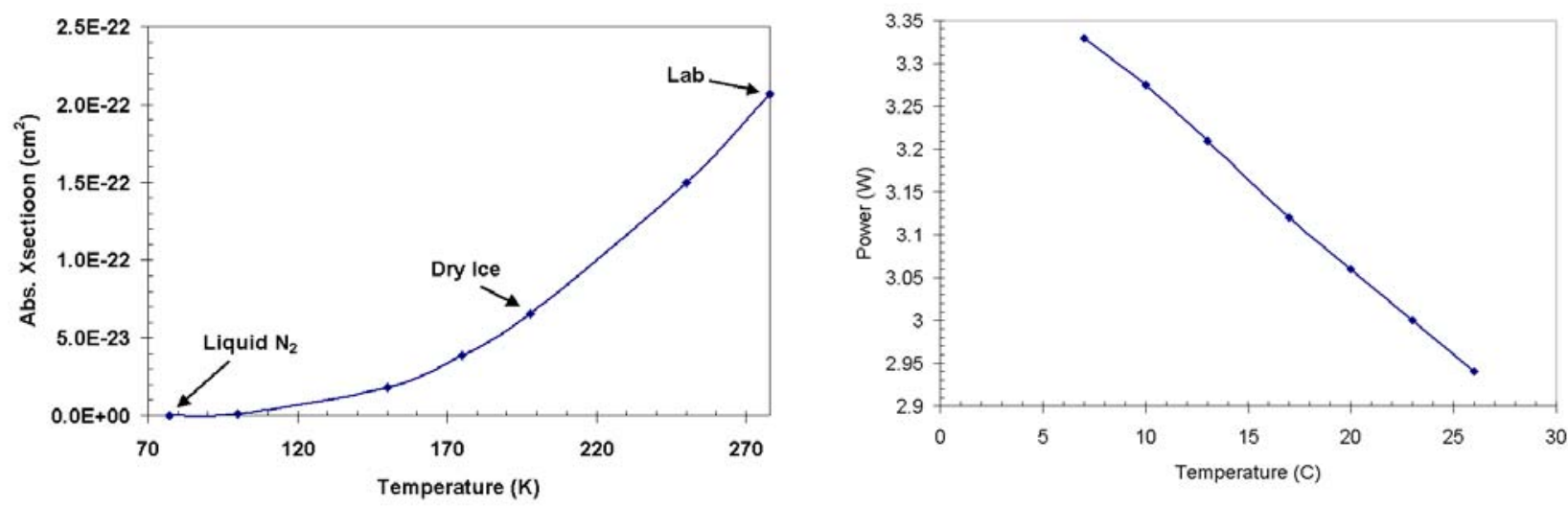

Figure 2: Left hand side (LHS): calculated absorption cross-section at $938 \mathrm{~nm}$ vs. temperature; right hand side (RHS): output power of first stage $938 \mathrm{~nm}$ fiber amplifier vs. temperature of copper heat sink for fiber coil.

The light at the output of the first amplifier stage has $\mathrm{M}^{2}<1.1$ and up to $3.5 \mathrm{~W}$ average power in continuous wave (nonpulsed) mode. The light from the first stage is collimated, passed through a quarter and half waveplate to adjust the polarization state, a short pass filter to eliminate $1088 \mathrm{~nm}$ ASE from propagating into the next stage and an optical 
isolator to prevent feedback into the first stage. The $3.5 \mathrm{~W}$ was measured at the output of the optical isolator after passing through all the filters and waveplates. The second stage was constructed from a $40 \mathrm{~m}$ piece of $\mathrm{Nd}^{3+}$ fiber with same core composition as the first stage fiber but with a $30 \mu \mathrm{m}, 0.06 \mathrm{NA}$ core and $125 \mu \mathrm{m}, 0.4 \mathrm{NA}$ polymer coated pump cladding. This fiber was again coiled to $8 \mathrm{~cm}$ diameter and taped to the inside of a water cooled copper pipe. The $20 \mu \mathrm{m}$ core in the first stage, when coiled to $8 \mathrm{~cm}$ effectively only propagated a single mode of the wave guide. The output of the laser diode was asymmetric and tended to excite higher order modes of the $30 \mu \mathrm{m}$ core fiber. It was difficult to find a precise bend radius for filtering out the higher order modes from the $30 \mu \mathrm{m}$ core. Thus the $20 \mu \mathrm{m}$ core fiber was chosen for the first stage, where it acted as a spatial filter ensuring single mode beam quality. This good quality beam was then launched into the $30 \mu \mathrm{m}$ core and propagated stably as a single spatial mode with an output $\mathrm{M}^{2}<1.2$ and good transmission $(>85 \%)$ through an optical isolator. The second stage was pumped with a $90 \mathrm{~W}$ fiber coupled laser diode with a $200 \mathrm{~mm}$ spot and $0.22 \mathrm{NA}$. This laser diode was coupled via lenses to the second stage fiber through dichroics that passed $808 \mathrm{~nm}$ and rejected $938 \mathrm{~nm}$ and $1088 \mathrm{~nm}$. The output of the second stage as a function of pump diode current $(40 \mathrm{~A} \rightarrow 90 \mathrm{~W})$ is shown in figure 3 (RHS) below. Total output power is shown along with power through a short pass filter (eliminating 1088nm ASE) and the optical isolator. We also show the total power incident on the frequency conversion crystal, which accounts for loss in the telescope that sets the beam size and location of the waist as well as the dichroic which combines the $938 \mathrm{~nm}$ and $1583 \mathrm{~nm}$ beams. In figure 3 (LHS) we show a picture of the fiber coiled inside its copper block heat sink.
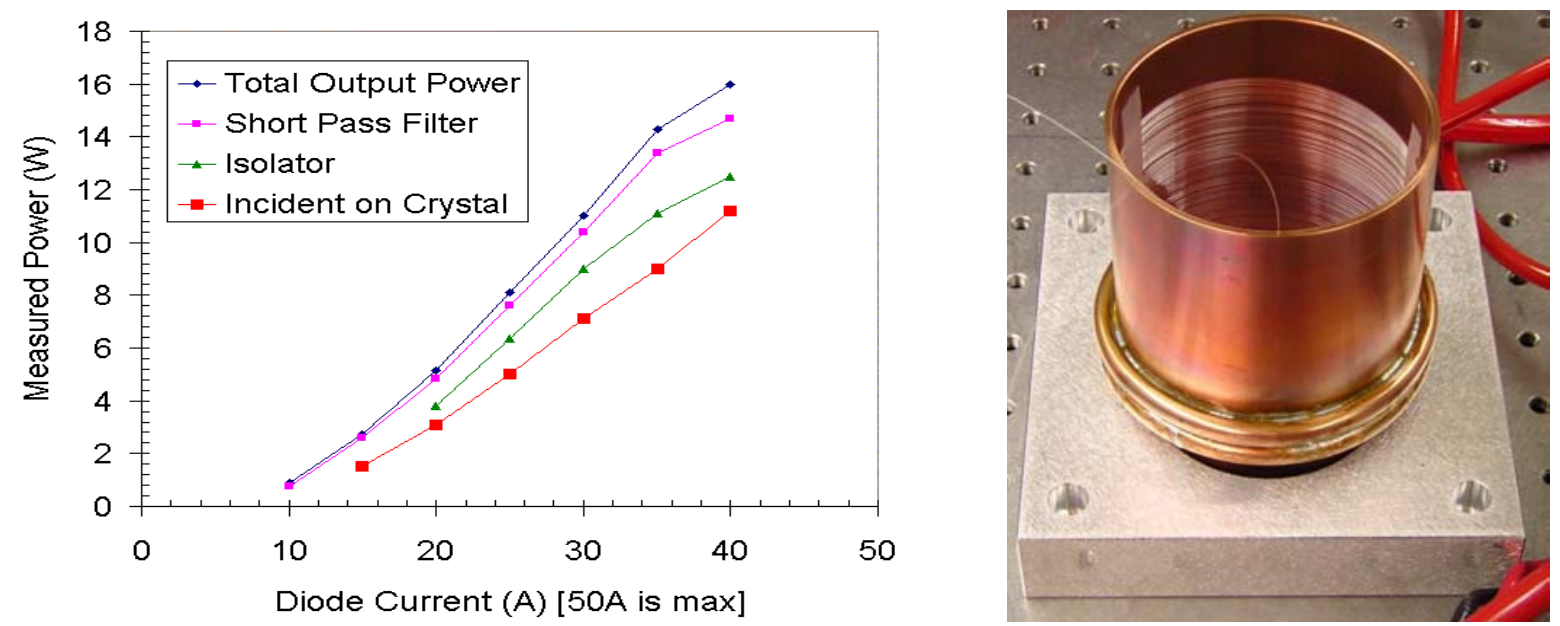

Figure 3: Right hand side (RHS): Power vs. diode pump current of second stage neodymium fiber amplifier. Left hand side (LHS): water cooled copper heat sink to maintain fiber at room temperature in the presence of a strong pump laser.

We have also investigated operation of this amplifier system in a pulsed format. First we sought to characterize the susceptibility of the system to stimulated Brillouin scattering (SBS). The response of fiber lasers to this effect is fairly well characterized at $1550 \mathrm{~nm}$ where it is critical to the telecom industry, but less well characterized at shorter wavelengths. To this end, we first measured the bandwidth of our $938 \mathrm{~nm}$ diode laser as a function of the RF noise generator power. Then we pulsed the light entering the fiber amplifier. We measured the input signal, output signal, and return signal. We know the fiber length, core size and signal line width. From this information we were able to determine the power level for the onset of SBS as a function of the signal line width using equations 1-3 below [9].

$$
P_{0}^{c r}=\frac{21 \cdot A_{e f f}}{g_{B}(\Delta v) \cdot L_{e f f}}
$$

where,

$$
L_{\text {eff }}=\frac{1}{\alpha} \cdot\left[1-e^{-\alpha \cdot L}\right]
$$


and,

$$
g_{B}(\Delta v)=\frac{g_{B}}{1+\frac{\Delta v}{\Delta v_{B}}}
$$

Here $\mathrm{P}_{0}^{\text {cr }}$ is the input power to the amplifier for the onset of SBS, $\mathrm{A}_{\text {eff }}$ is the effective area of the core (which we take to be $\pi$ times the mode field radius squared), $\mathrm{L}$ is the length of the amplifier, a is the loss or gain of the amplifier in nepers, $\Delta v$ is the bandwidth of the signal laser and $\mathrm{g}_{\mathrm{B}}$ is the Brillouin gain and $\Delta v \mathrm{~B}$ is the Brillouin gain bandwidth. The latter two parameters are the fit parameters. In practice, the input power was held constant and the gain of the amplifier was increased until SBS was observed. The bandwidth, measured output power at the onset of SBS, the theoretical fit and fit parameters from this experiment are shown in table 1 below.

\begin{tabular}{|l|l|l|}
\hline $\begin{array}{l}\text { Bandwidth } \\
(\mathrm{MHz})\end{array}$ & $\begin{array}{l}\text { Measured } \\
\text { Output } \\
\text { Power }(\mathbf{W})\end{array}$ & $\begin{array}{l}\text { Theoretical } \\
\text { Output } \\
\text { Power }(\mathbf{W})\end{array}$ \\
\hline 10 & 18.84 & 18.835 \\
\hline 20 & 19.78 & 19.864 \\
\hline 50 & 22.6 & 22.448 \\
\hline 160 & 33.1 & 32.136 \\
\hline 310 & 47.3 & 46.245 \\
\hline
\end{tabular}

\begin{tabular}{|l|l|l|}
\hline & Units & $\begin{array}{l}\text { Theoretical } \\
\text { Fit to data }\end{array}$ \\
\hline$g_{B}$ & $\mathrm{~m} / \mathrm{W}$ & $4 \times 10^{-11}$ \\
\hline$\Delta v_{B}$ & $\mathrm{MHz}$ & 310 \\
\hline
\end{tabular}

Table 1: Left hand side: measured data from SBS experiment and theoretical fit to the data. Right hand side: fit parameters used to construct the theoretical output power in the last column on the right hand side table. All data was taken at $938 \mathrm{~nm}$.

Using this knowledge, we have begun investigating the $938 \mathrm{~nm}$ laser in pulsed formats. To date, we are operating at $100 \mathrm{kHz}$ repetition rates so that square pulse distortion is minimal. Output data from the system in essentially the same configuration as was used to generate the $\mathrm{CW}$ data set seen in figure 3 above is shown in figure 4 below.

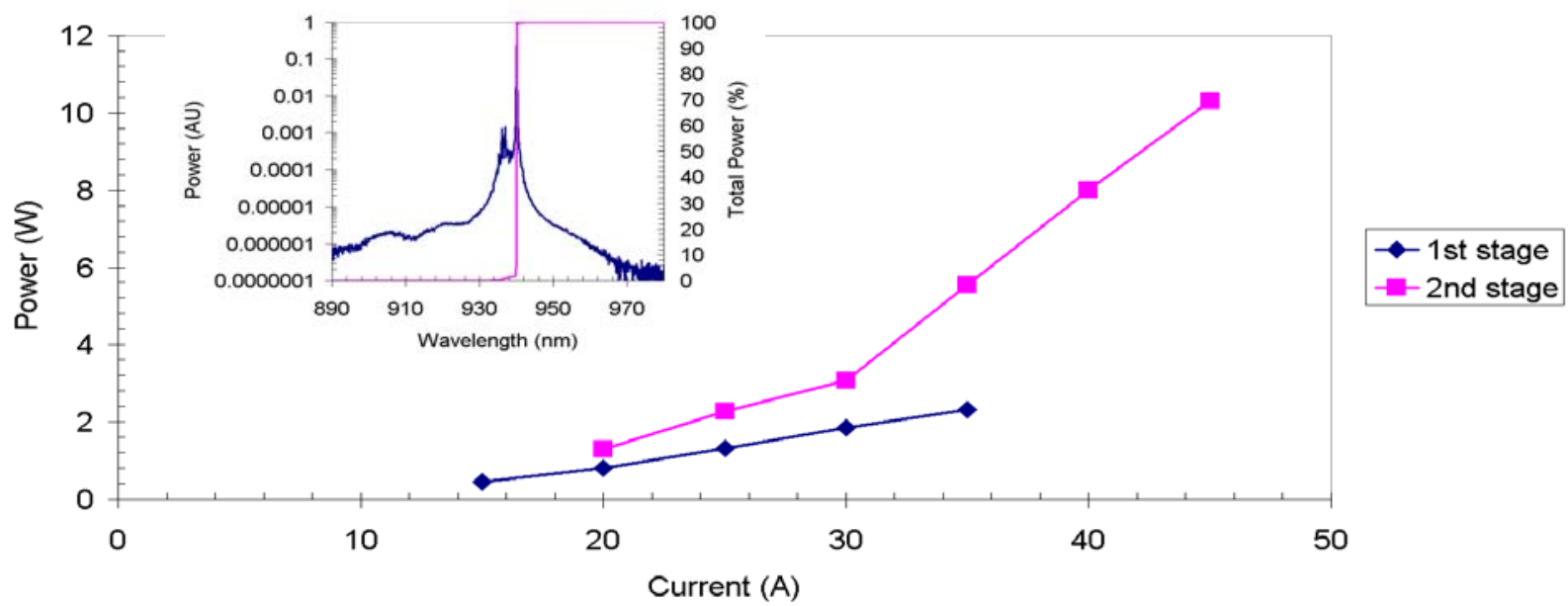

Figure 4: Power vs. pump diode current of $1^{\text {st }}$ and $2^{\text {nd }}$ stage fiber amplifiers in pulsed operation. Inset: spectra at amplifier output and integration of spectra showing $>95 \%$ of the power is at the signal wavelength. 
In figure 4 , the laser was pulsed at $100 \mathrm{kHz}$ with a $20 \%$ duty cycle (square waveform) and the signal line width was $500 \mathrm{MHz}$. $10 \mathrm{~W}$ of total output power was achieved. A spectral measurement (insert) shows that $>95 \%$ of the optical power measured is at $938 \mathrm{~nm}$. Output power from the first stage was deliberately limited to $1.4 \mathrm{~W}$ or about $17 \mathrm{~dB}$ of gain at $938 \mathrm{~nm}$. At higher gain the first stage began to show signs of Q-switching due to gain buildup at $1088 \mathrm{~nm}$. Higher duty cycles performed equally or better than this. $10 \%$ duty cycle ran into problems with low signal power (due to limited gain per stage required to prevent Q-switching at $1088 \mathrm{~nm}$, we cannot just turn up the gain per stage) and an additional stage of amplification will be necessary to operate in this regime. However, the SBS modeling indicates that duty cycles down to $5 \%$ are feasible at $10 \mathrm{~W}$ average power levels.

\section{ERBIUM FIBER LASER AT 1583 NM}

Our $1583 \mathrm{~nm}$ fiber laser system employs a Koheras DFB single frequency fiber laser as the master oscillator. This unit is several years old and is not polarization maintaining or very high power $(\sim 1-2 \mathrm{~mW})$ an upgrade is in progress to address these issues. The light from the master oscillator is transmitted through an optical isolator and then is sent to a single mode pre-amplifier made from a single mode WDM, a $500 \mathrm{~mW} 980 \mathrm{~nm}$ pump laser, about $50 \mathrm{~m}$ of single mode erbium fiber, an optical isolator and a polarization controller. It is then transmitted through a lithium niobate phase modulator where it its bandwidth is increased to $500 \mathrm{MHz}$ by driving the phase modulator at $100 \mathrm{MHz}$ at several time $\mathrm{V}_{\mathrm{pi}}$. The phase modulated light is sent into a 90/10 PM fiber splitter with a polarizer on the $10 \%$ output that can be monitored with a power meter. Light through the polarizer is maximized using the polarization controller at the output of the pre-amplifier. The light from the $90 \%$ port is launched into a 14W IPG PM fiber amplifier (model \# EAR-15k1583-LP-SF). This module outputs a $5 \mathrm{~mm}$ collimated beam, which we down telescope to the size required for our frequency conversion system after which the light is sent through a bulk optical isolator. We have also run this laser system as a pulsed laser by inserting an additional polarization controller and a PM lithium niobate amplitude modulator prior to the pre-amplifier. However, as the seed laser is not polarization maintaining and the lithium niobate phase modulator is polarization sensitive, the extinction ratio of the modulation is relatively poor $(<10 \mathrm{~dB})$. Again we are pursuing an upgrade of the front end of this laser system, which we believe will make it more useful as a pulsed laser in the near future.

Power vs. pump current and spectral output of the $1583 \mathrm{~nm}$ laser system run $\mathrm{CW}$ at $14 \mathrm{~W}$ is shown in figure 5 below.
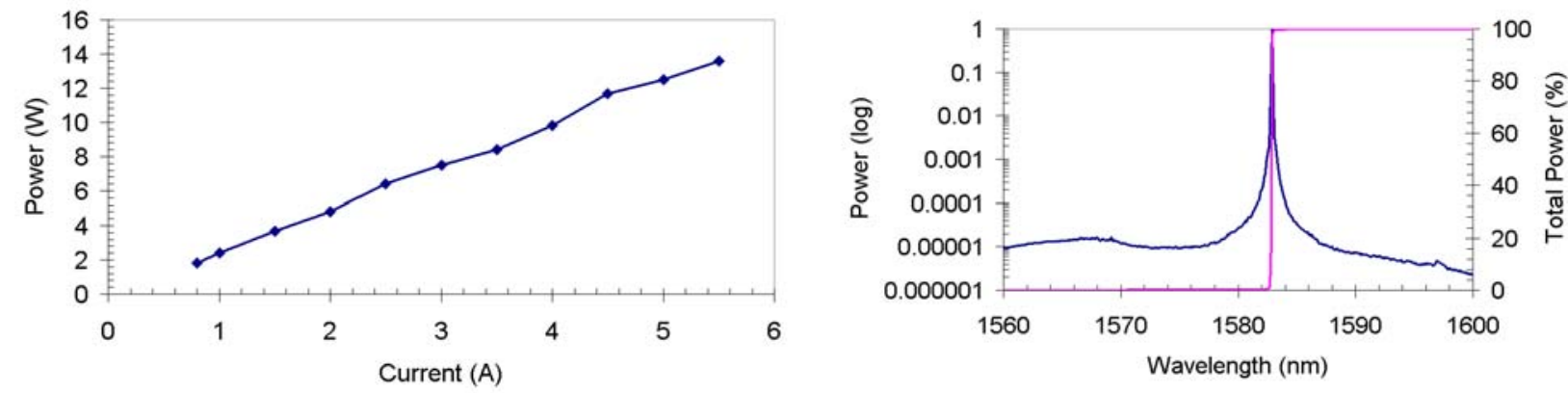

Figure 5: Left hand side: laser output power vs. pump laser current of the $1583 \mathrm{~nm}$ laser system run in CW mode. Right hand side: spectral output of the laser at 14W. The pink "total power" line is the ratio of the power up to that wavelength to the total integrated power contained in spectra expressed as a percentage.

$\mathrm{M}^{2}$ of the laser system was measured with a Coherent mode-master to be 1.05 . The spectrum shows that $>98 \%$ of the output power is at the signal wavelength. We also tested the laser system run in a pulsed format. For this case, we ran the laser with signal modulated in a square waveform at $20 \%$ duty cycle and $100 \mathrm{kHz}$ repetition rate. At $4 \mathrm{~A}$ pump current the laser emitted $10 \mathrm{~W}$ of average power with $95 \%$ of the power at the signal wavelength. However, the peak power of the modulated signal was only about $3 / 5^{\text {th }}$ of the expected value due to the poor extinction ratio of the modulator resulting from the issues with the polarization of the front-end laser. 


\section{SUM FREQUENCY MIXING}

The $938 \mathrm{~nm}$ and $1583 \mathrm{~nm}$ lasers were sum frequency mixed in periodically poled materials. We examined both PPKTP and PPSLT. Sum frequency mixing is slightly more challenging than frequency doubling. This is because there are two independent beams with different wavelengths. The beams need to be co-linear throughout the frequency conversion crystals. As they are being focused through the crystals this means that not only do they need to be co-linear at the lens but that they need to have the same confocal parameter and are focused to the same location in the focused region. Colinearity is assured in our system by having 2 alignment irises about $1.5 \mathrm{~m}$ apart on the table. By ensuring that each beam is well centered on both irises, we can assure the two beams are co-linear $<1 \mathrm{mrad}$. It is more difficult to ensure they have the same focus and confocal parameter.

Optimum conversion efficiency is achieved for Boyd-Klienman focusing [10] which indicates the crystal length should be about 2.7 times the confocal parameter. The confocal parameter is a function of the beam wavelength and diameter. An achromatic lens was used to make sure the beams focused to the same position. To ensure the beams have the same confocal parameter the beam diameters need to be set to a ratio of 0.77 . The latter condition was established by using our Coherent Mode Master to measure the beam diameter and location of the beam waist at the position on the table where we planned to put the focusing lens. Collimating telescopes were employed to adjust the diameter of the beams to the right size and to locate the beam waists at the focusing lens. This turns out to be a rather tedious process in practice. The Mode Master was very helpful in this endeavor in that it could quickly measure the beam diameter, divergence and location of the beam waist and beam waist diameter relative to the front plane of the instrument. We set the $1583 \mathrm{~nm}$ beam to $2.0 \mathrm{~mm}$ diameter with the waist within $50 \mathrm{~cm}$ of the focusing lens and the $938 \mathrm{~nm}$ beam was set to $1.5 \mathrm{~mm}$ diameter with the waist within $50 \mathrm{~cm}$ of the focusing lens. A $75 \mathrm{~mm}$ focal length lens was then employed to focus the beams into our periodically poled materials both of which were $3 \mathrm{~cm}$ long. We calculated that this lens should yield Boyd-Klienman focusing in our crystals and indeed we tried a $60 \mathrm{~mm}$ and $90 \mathrm{~mm}$ focusing lens and the peak conversion efficiency was achieved at $75 \mathrm{~mm}$. A Pellan Broca prism is used at the output of the crystals to separate the $589 \mathrm{~nm}$ light from the IR light prior to detection with a power meter.

In CW mode the two lasers produced $2.7 \mathrm{~W}$ of $589 \mathrm{~nm}$ light in PPKTP. The $589 \mathrm{~nm}$ power vs. total combined IR power curve is shown in figure 6 below. Above $2.7 \mathrm{~W}$ the power rolled off and there was evidence of crystal degradation. This compares favorably with our prior results for frequency doubling the $938 \mathrm{~nm}$ laser to $469 \mathrm{~nm}$ in which PPKTP showed similar signs of damage in similar focusing conditions at about $0.5 \mathrm{~W}$ of $469 \mathrm{~nm}$ power. We attempted pulsing the $938 \mathrm{~nm}$ system and $1583 \mathrm{~nm}$ system at $20 \%$ duty cycle, which should yield a $5 \mathrm{X}$ increase in converted power. The converted power increased at low IR power by a factor of about $3 \mathrm{X}$, however the power still rolls off at about $2.5 \mathrm{~W}$. So while fewer IR photons are used no more photons are generated at $589 \mathrm{~nm}$ where we want them. The right hand side of figure 6 shows the oven, Pellan Broca and a sodium cell fluorescing with the yellow glow from the 589nm light.
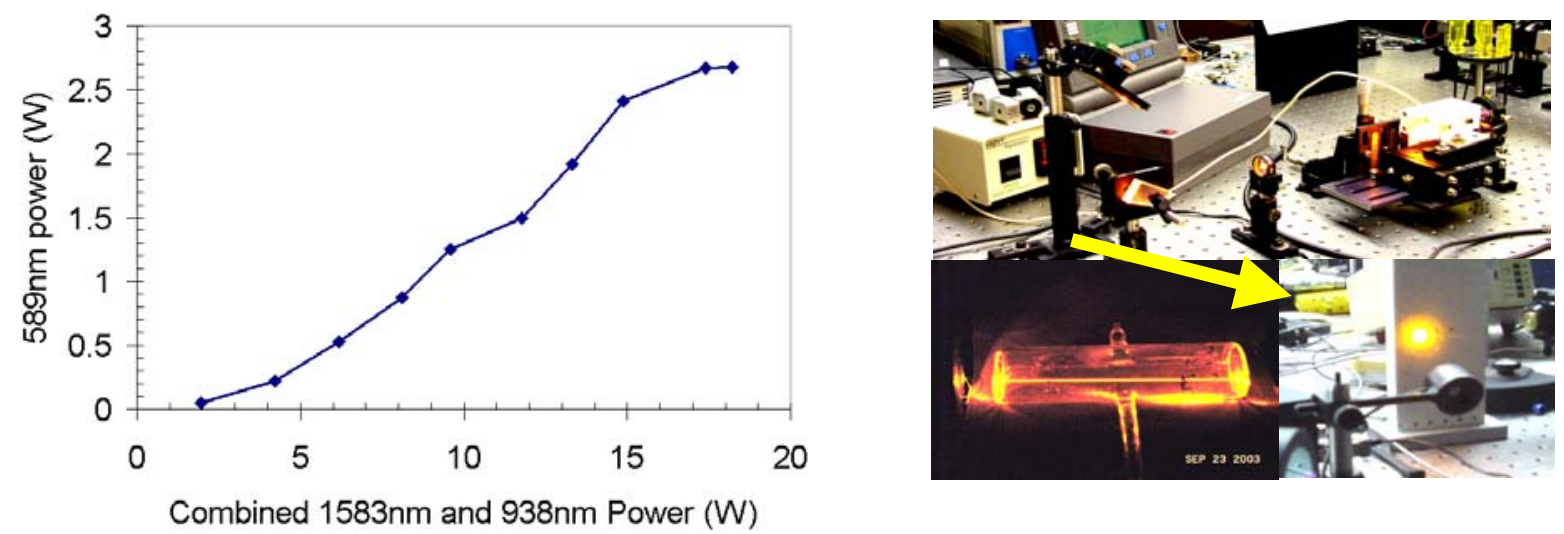

Figure 6: Left hand side: $589 \mathrm{~nm}$ power vs. combined $1583 \mathrm{~nm}$ and $938 \mathrm{~nm}$ power for Boyd Kleinman focusing in a $3 \mathrm{~cm}$ long PPKTP crystal. Right hand side: pictures of $589 \mathrm{~nm}$ light from the experiment, oven, collimating lens and PellanBroca prism (top), fluorescence of the $589 \mathrm{~nm}$ beam in a sodium vapor cell (bottom left), low power $589 \mathrm{~nm}$ spot on a beam stop (lower right). 
The PPSLT crystal, poled by Physical Sciences Incorporated yielded superior results. Again a $3 \mathrm{~cm}$ crystal was employed using the same beam and focusing conditions described above for the PPKTP crystal. In the CW case the PPKTP had slightly conversion efficiency. However, when we ran the system at $20 \%$ duty cycle, we were able to achieve $3.5 \mathrm{~W}$ of output power (figure 7 below).

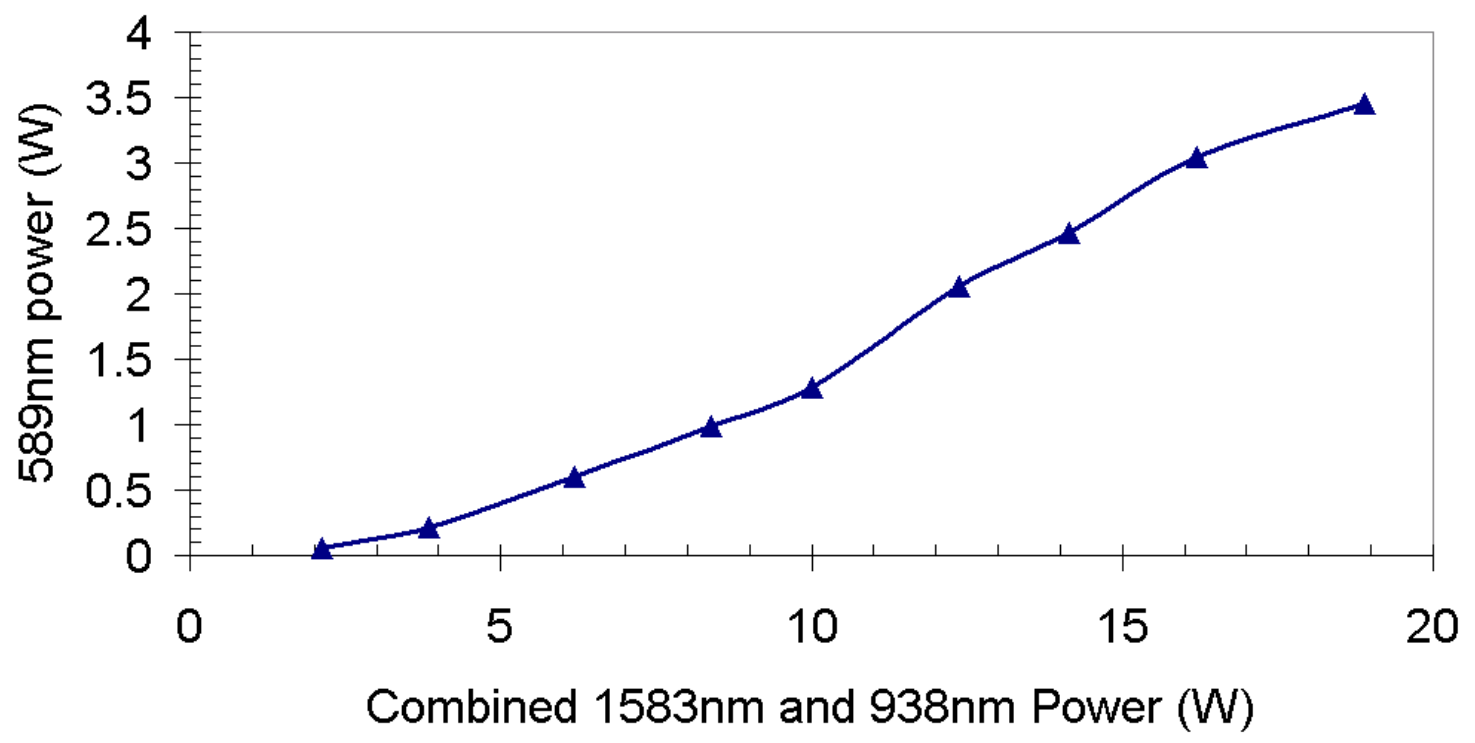

Figure 7: PPSLT results of $589 \mathrm{~nm}$ power vs. combined $1583 \mathrm{~nm}$ and $938 \mathrm{~nm}$ power.

Again, about a $3 \mathrm{X}$ increase in converted power over the $\mathrm{CW}$ case was achieved. This slightly lower than the $5 \mathrm{X}$ expected increase for this duty cycle. This discrepancy is a result of the issues discussed above concerning the $1583 \mathrm{~nm}$ laser modulation extinction due to the polarization issues. At low $1583 \mathrm{~nm}$ power $(\sim 1 \mathrm{~W})$ we have achieved a $5 \mathrm{X}$ increase in conversion over the $\mathrm{CW}$ case as expected by careful tweaking of the polarization controllers. However the system was not terribly stable at this optimized operating point. As such, it was decided not to run the $1583 \mathrm{~nm}$ laser higher in power at the optimized operating point prior to receiving the components we have ordered for the front-end upgrade. There was no sign of damage in the PPSLT at the $3.5 \mathrm{~W}$ level over the timeframe of the experiment, which was albeit somewhat short ( 30min). We believe the PPSLT crystal will permit power scaling to 5-10W average power at $589 \mathrm{~nm}$.

\section{CONCLUSIONS}

We are developing $\mathrm{CW}$ and pulsed 589nm fiber lasers for sodium laser guide star applications. We have achieved $>15 \mathrm{~W}$ of power at $938 \mathrm{~nm}$ with a $\mathrm{Nd}^{3+}$ based fiber amplifier system. We have measured the SBS gain and line width of our $\mathrm{Nd}^{3+}$ amplifier fiber to be $4 \times 10^{-11} \mathrm{~m} / \mathrm{W}$ and $310 \mathrm{MHz}$ respectively. We have demonstrated laser operation at $100 \mathrm{kHz}$ repetition rates with duty cycles as low as $20 \%$ and believe $5-10 \%$ duty cycles are possible, based on our SBS measurements. We have constructed a $14 \mathrm{~W} 1583 \mathrm{~nm}$ laser system from commercial components. This laser system is capable of operating in a pulsed format, but requires an improved, all PM front-end system to do this well. This latter upgrade to the system is in progress. We have achieved $3.5 \mathrm{~W}$ of $589 \mathrm{~nm}$ light via sum frequency mixing in a PPSLT crystal. We saw no signs of optical damage in the crystal during the course of our test.

This work was performed under the auspices of the U.S Department of Energy by the University of California, Lawrence Livermore National Laboratory under Contract No. W-7405-Eng-48

We would like to acknowledge the generous support of the NSF Center for Adaptive Optics (CFAO), NSF/AURA Adaptive Optics Development Program (AODP) and LLNL's Laboratory Directed Research and Development (LDRD). 
This material is based upon work supported, in part, by the US Air Force Research Laboratory Directed Energy Directorate under Contract No.F29601-03-C-0044. Any opinions, findings, conclusions or recommendations expressed in this material are those of the authors and do not necessarily reflect the views of the US Air Force.

\section{REFERENCES}

1. D. M. Pennington, "Laser guided adaptive optics for high resolution astronomy," CLEO 2002, paper CMN5

2. D. Bonaccini, W.K. Hackenberg, M. J. Cullum, E. Brunetto, T. Ott, M. Quattri, E. Allaert, M. Dimmler, M. Tarenghi, A. Van Kersternen, C. Di Chirico, B. Buzzoni, P. Gray, R. Tamai, M. Tapia, "ESO VLT laser guide star facility," Proceedings of the SPIE, vol. 4494, pp. 276-289, (2002)

3. J.C. Bienfang, C.A. Denman, B.W. Grime, P.D. Hillman, G.T. Moore, J.M. Telle, "20W of continuous wave sodium D2 resonance radiation from sum-frequency generation with injection locked lasers," Optics Letters, vol.28, no. 22, pp. 2219-2221, (2003)

4. A.J. Tracy, A.K. Hankla, C.A. Lopez, D.C. Sadighi, N. Rogers, K. Groff, I. T. McKinnie, C. d"Orgeville, "High power solid state sodium beacon laser guide star for the Gemini North Observatory," Proceedings of the SPIE, vol. 5490, pp. 998-1009 (2004)

5. V. Velur, E. J. Kibblewhite, R.G. Dekany, M. Troy, H.L. Petrie, R.P. Thicksten, G. Brack, T. Trin, M. Cheselka, "Implementation of the Chicago sum frequency laser at Palomar laser guide star test bed," Proceedings of the SPIE, vol. 5490, pp. 1033-1040 (2004)

6. S.A. Payne, et al, "Synthetic guide star generation," US Patent \# 6,704,331

7. P. Dragic and G. Papen, "Efficient amplification using the ${ }^{4} \mathrm{~F}_{3 / 2}{ }^{4} \mathrm{I}_{9 / 2}$ transition in Nd-doped silica fiber," IEEE Phot. Tech. Lett. 11, 1593-1595 (1999)

8. J.W. Dawson, R. J. Beach, A. Drobshoff, Z.M. Liao, D.M. Pennington, S.A. Payne, L. Taylor, W. Hackenberg, D. Bonaccini, presented at Advanced Solid State Photonics, Santa Fe, NM, 2004

9. G.P. Agrawal, "Non-linear fiber optics, $2^{\text {nd }}$ Edition", Academic Press 1995

10. G.D. Boyd and D.A. Kleinman "Parametic Interaction of Foucsed Gaussian Light Beams", J. of Appl. Phy., Vol. 39, No. 8, 3597-3639, July 1968 